\title{
The Frequency of Emotions and Emotion Variability in Self- regulated Learning: What Matters to Task Performance?
}

\author{
Shan Li, Juan Zheng \& Susanne P. Lajoie ${ }^{1}$ \\ Department of Educational and Counselling Psychology, McGill University, Montreal, QC, Canada \\ Article received 30 June 2021 / Article revised 4 September 2021 / Accepted 17 October / Available online 5 November
}

\begin{abstract}
Emotion variability and its relationship to performance is an underexplored area of research both inside and outside the realm of medical education. We address this gap by examining the relative importance of the frequency of emotions and emotion variability that occurred in specific phases of self-regulated learning (SRL) in predicting students ' performance. Specifically, 23 medical students were recruited to complete the task of diagnosing a virtual patient in a hospitalsimulated environment. Students' facial expressions were video-recorded and were classified into basic emotions. We calculated the frequency of emotions and emotion variability at each SRL phase: forethought, performance, and selfreflection. Findings revealed that both the frequency of emotions and emotion variability influenced clinical reasoning performance, but they functioned differently in different SRL phases. Moreover, emotion variability negatively predicted performance regardless of which SRL phases it was tied to. This study helps shift the focus of research from the effect of emotions on performance to the joint effect of emotion and emotion variability, which has the potential to address the inconsistency in emotion-related research findings. Although we situate the study in the context of clinical reasoning, findings from this research inform the research of emotion in learning and instruction for other domains. Furthermore, this study lays the foundation for future advances in emotion-related study designs since the introduction of emotion variability leaves many questions unanswered and shows promise for new research directions.
\end{abstract}

Keywords: Emotion; Emotion Variability; Self-regulated Learning; SRL phases

\footnotetext{
${ }^{1}$ Corresponding author: Susanne P. Lajoie, Department of Educational and Counselling Psychology, McGill University, Canada, email address: susanne.lajoie@mcgill.ca ;DOI:https://doi.org/10.14786/flr.v9i4.901
} 


\section{Introduction}

Medical students experience a range of emotions in clinical settings. For instance, they may experience surprise during the diagnoses of some patients with unexpected symptoms; they may experience joy and even relief when a correct diagnosis is reached, or; they may worry about their patient's emotions and expectations when given bad news. These emotions, and others, undoubtedly affect a medical student's thoughts, behaviours, and performance. Given that emotions change over time, an inherent attribute of emotion is emotion variability (Barrett, 2009), which is defined as the fluctuations in emotional states (Oliver \& Simons, 2004). For instance, two medical students may have the same level of positive emotions but differ from one another in their emotion variability, with one student changing his or her emotions frequently and the other person changing such emotions rarely. Research on the role of emotions in clinical reasoning is still nascent, but it is beginning to draw more attention from medical education researchers (Artino, Holmboe, \& Durning, 2012; Lajoie, Zheng, \& Li, 2018; McConnell et al., 2016). Nevertheless, in a review of the medical education literature, no research has been conducted on the relationship between emotion variability and student ability to diagnose patients. Therefore, it is necessary to develop an understanding of how emotion and emotion variability jointly affect students' performance. The purpose of this study is to examine the roles of emotion and emotion variability in clinical reasoning and to compare their relative importance in predicting diagnostic performance. Although we situated this study in the context of clinical reasoning, this study informs future research on the role of emotion variability in learning and instruction across different disciplines. Emotion variability and its relationship to performance is an underexplored area of research both inside and outside the realm of medical education (Gruber, Kogan, Quoidbach, \& Mauss, 2013). Moreover, this study takes the dynamic aspect of emotions (i.e., emotion variability) into account, which has the potential to address the inconsistencies in emotion-related research findings. Lastly, this study presents new research directions by assessing the relative importance of the many aspects of emotions.

\section{Theoretical rationale}

\subsection{Emotion-related research}

Research on emotions has flourished in various disciplines and contexts, as reflected in the abundant classification of emotions distinguishing between basic emotions, achievement emotions, epistemic emotions, and social emotions, as well as the emergence of numerous advanced emotion detection techniques (Izard, 2007; Pekrun \& Stephens, 2010). According to Ekman (1992), basic emotions (anger, disgust, fear, happiness, sadness, and surprise) are innate, universal, automatic, and fast neurological and behavioural responses to environmental challenges. For this paper, we limit our exploration to basic emotions since they are universal and may function as building blocks for more complex emotions. In addition, basic emotions are likely to be activated in medical settings that are by nature high-stakes (Lajoie, Zheng, Li, Jarrell, \& Gube, 2019; Tracy \& Randles, 2011). Furthermore, basic emotions can be captured in situ as behavioural measures (e.g., facial expression detection) provide an unobtrusive real-time emotional expression trace (Lajoie et al., 2019). We operationalized basic emotions by their frequency as they occurred in situ since the frequency of emotions has been extensively investigated in various contexts (Barrett, 2006). Specifically, the frequency of emotions refers to how often a person experiences a dominant affect. The literature regarding the relationship between the frequency of emotions and performance is mixed (McConnell et al., 2016). It is our contention that the frequency of emotions alone does not provide a complete picture of how emotions affect learning and performance. In this study, we make an effort to examine the joint effect of emotion (i.e., the frequency of emotions) and emotion variability (i.e., how much it varies) on problem-solving 
performance to advance a complete understanding of how different aspects of emotion influence diagnostic performance.

\subsection{Emotion Variability}

Emotion variability represents the dynamic nature of emotion above and beyond what researchers can gain from exploring the overall frequency of emotion. Appraisal approaches to emotion, which proposes that emotions arise from a meaningful analysis of a situation, account for variability in emotional responses (Scherer, 2005). Moreover, some researchers argued that emotion variability is an inherent attribute of emotion since each emotion remains more or less constant over time (Barrett, 2009). As of yet there are no empirical studies of the influence of emotion variability on clinical reasoning; however, there are studies from other fields, such as psychological health, that present two competing perspectives on whether emotion variability impedes or promotes performance (Gruber et al., 2013). One perspective argues that greater variability in emotions is related to poorer outcomes (Thompson, Boden, \& Gotlib, 2017) since substantial changes in emotions deplete an individual's mental resources and passions (Xu, Martinez, Van Hoof, Eljuri, \& Arciniegas, 2016). The other perspective contends that emotion variability has a positive influence on outcomes, especially students' wellbeing (Kashdan \& Rottenberg, 2010). Researchers who hold this latter perspective consider emotion variability as adaptive in that individuals demonstrate that they can respond adaptively to changing environments or task conditions. Our study can add new empirical evidence that would shed light on these competing perspectives.

\subsection{Emotion and self-regulated learning}

While there is a pressing need to examine the effect of emotion and emotion variability simultaneously in one study, it is noteworthy that such examinations should be situated in the context of human thinking and learning, which by its nature, is guided by contemporary learning theories (Artino et al., 2012). Self-regulated learning (SRL) is a well-established learning theory that provides researchers with a theoretical framework to study how students regulate their behaviours, cognition, motivation, and emotion to achieve personal goals (Li \& Lajoie, 2021; Pintrich, 2000; Zimmerman, 2000). According to Zimmerman (2000), SRL consists of three phases: forethought, performance, and self-reflection. The three phases are structurally interrelated and cyclically sustained in students' learning process. In the forethought phase, students analyse the task, set personal goals, and plan appropriate strategies for the attainment of their predetermined goals. While the forethought phase is a preparation step for SRL, the performance phase is an actual executing process in which students control and monitor their learning activities. The self-reflection phase involves students' self-judgement and reaction to the performance. As learners progress through these three phases, their performance yields specific emotions. Emotions can serve to sustain and moderate the SRL processes, or they may hinder such processes, which could significantly affect learning outcomes (Lajoie et al., 2018; Pekrun \& Stephens, 2010). Instead of viewing emotion as a by-product of SRL, Ben-Eliyahu (2019) considered emotions as an important target of learning, i.e., achieving emotional wellbeing in learning or problemsolving. On that account, Ben-Eliyahu (2019) proposed an academic emotional learning (AEL) cycle to understand how learners actively monitor and modify their emotions in SRL using various self-regulated emotion (SRE) strategies. In line with predominant SRL models, SRE occurs in "a weakly sequenced recursive cycle" (Ben-Eliyahu, 2019, p 92), in which earlier emotional experiences update conditions on which a student experiences a subsequent emotion. Emotions can and should be examined in the phases of SRL in a similar way to that of behaviours, cognitive and metacognitive activities.

As illustrated in the modal model of emotion (Gross, 2013), a psychologically relevant situation triggers focused attention from individuals to assess the meaning of that situation in light of relevant goals and consequently generates emotional responses. As person-situation transactions unfold over time, emotion fluctuates in learning and problem-solving, depending on how individuals perceive and 
assess the evolving situation (John \& Gross, 2006). According to Schutz and Davis (2000), the forethought phase is a preparatory phase whereby students determine how to best prepare for a task and how to actually prepare for the task. Therefore, the most prevalent emotions during this phase are anticipatory emotions (e.g., anxiety, distress, hope, or fear) that are triggered by a perceived threat or challenge. The forethought phase is "a period of mixed emotions" (Schutz \& Davis, 2000, p. 249). In the performance phase, students' emotions are affected by their subjective judgement of the task, the students' level of preparation, and perceived ability to solve any potential problems that might occur during the task. There has been a tremendous amount of research examining the emotion of anxiety in testing situations in the phase of performance; however, few studies have documented students' frequent emotions while solving real-world problems (Pekrun et al., 2002). The emotions that are most prevalent in the self-reflection phase tend to be either harmful (e.g., disappointed) or beneficial (e.g., happy), depending on how well students performed the task and the attributions they made about the performance (Schutz \& Davis, 2000). The interplays between emotions and SRL strategies (e.g., evaluation and monitoring) are beginning to receive attention from researchers (Ahmed, van der Werf, Kuyper, \& Minnaert, 2013; Lajoie et al., 2019). For instance, Lajoie et al. (2019) examined the differences in the co-occurrence of emotions and SRL strategies between high and low performing medical students as they diagnosed a virtual patient. Ben-Eliyahu and Linnenbrink-Garcia $(2013,2015)$ investigated emotion regulation from within the SRL perspective and its relationship with SRL strategies and learning achievement. Nevertheless, the relationships between the construct of emotion variability, SRL phases, and learning performance have been under-researched.

\subsection{The current study}

The relationship between the valence of emotions (i.e., positive versus negative) and performance is starting to be addressed in the context of clinical reasoning (Artino, Hemmer, \& Durning, 2011; Artino et al., 2012; Harley et al., 2015; Lajoie et al., 2018, 2019; McConnell \& Eva, 2012). It is noteworthy, however, that positive emotions are not always beneficial and negative emotions are not always detrimental (Pekrun \& Stephens, 2010). For instance, anxiety initially increases one's vigilance of a task situation whereby they allocate more resources on the task to perform well. Therefore, it may generate more consistent results when examining the relationships between students' performance and the frequency of emotions rather than different categories of discrete emotions. There are also several other considerations that have led us to focus on the frequency of emotions instead of discrete emotions. First, students' emotional experiences could be better described by the frequency of emotions, given that students may experience a mixed emotional state at a certain time. Moreover, there is extensive literature on the relationships between discrete emotions and performance. We contend that the frequency of emotions may provide additional insights into how the features of emotions predict performance. Furthermore, examining the frequency of emotions minimizes the influences of individual differences since students respond to even the same situation with different emotions (Siemer, Mauss, \& Gross, 2007). In addition, we argue that emotion variability plays a role in determining students' performance. To the best of our knowledge, few attempts have been made in the literature to investigate emotion variability in the context of clinical reasoning. In particular, this study is one of the first to examine how the frequency of emotions and emotion variability in different SRL phases contribute to students' diagnostic performance simultaneously. This study is exploratory in nature; as such, we cannot propose any specific hypotheses. In sum, this study aims to address this gap by answering the following research questions: do the frequency of emotions and emotion variability affect students' performance in clinical reasoning? If so, what is the relative importance of the frequency of emotions and emotion variability that occurred in specific SRL phases in predicting performance? 


\section{Methods}

\subsection{Participants}

Considering that medical students are unique in that medical schools are highly selective, one consideration for determining the sample size is the eligible participants we are able to recruit. In addition, the number of samples should not be smaller than the number of variables since we used the assessment method of Averaging over Orderings to compare the relative importance of predictor variables $(\mathrm{Bi}, 2012)$. We described the method of Averaging over Orderings below. As a result, we recruited 23 medical students (10 males and 13 females) in a large public research university in Canada. The students were in their second year of medical study, with an average age of $24.8(S D=3.97)$. They had completed a 7-week module on Endocrinology, Metabolism, and Nutrition. Therefore, the participants shared a similar level of knowledge on the problem-solving scenarios that were designed specifically for this study. It is important to note that we had six predictive variables in this study, i.e., the frequency of emotions and emotion variability in each of the three SRL phases. Our sample size was three times larger than the number of the predictive variables, indicating the sample size of this study was adequate.

The study was approved by the local Research Ethics Board (REB) office and consent forms made students aware of the general purpose, procedures, and data collection processes (e.g., their facial expressions would be recorded) of this study. Students stated that they felt comfortable diagnosing virtual patient cases in technology-rich environments. Participants had the option to withdraw their consent and discontinue their involvement at any time. All participants completed the study; however, the emotional data of two participants were not used due to technical problems.

\subsection{Task and learning context}

Students were tasked with diagnosing one virtual patient case (i.e., Amy case) in BioWorld (Lajoie, 2009). BioWorld is a simulation platform that helps medical students practice clinical reasoning skills. As shown in Figure 1, students were first presented with a description of the patient's profile and symptoms, whereby they collected useful evidence for their diagnosis. They were required to formulate diagnostic hypotheses and to confirm or disconfirm their hypotheses by re-reading the case description, ordering lab tests, or searching the library within the system. In particular, clinical laboratory test results are important parameters in the decision-making process of diagnosis, and BioWorld system provides a full list of laboratory tests such as urine and blood tests. The online library that is embedded in the platform provides students information on diseases and corresponding diagnostic procedures; hence, students with varying levels of declarative knowledge get support based on their needs. It is worth mentioning that students can store the results of laboratory tests or searching results in the evidence palette for future reference. In essence, the evidence palette provides a mechanism for students to monitor what and how much information they have gathered for diagnosis. After submitting a final hypothesis, students engage in the self-reflection phase of problem-solving. They check the relevance of collected evidence items and lab tests to their final hypothesis, indicating which evidence items and lab tests are neutral to, support or against their diagnosis. In addition, students are asked to rank evidence items and lab tests based on their importance to the final hypothesis. They end the task by writing a case summary of their clinical reasoning processes. 


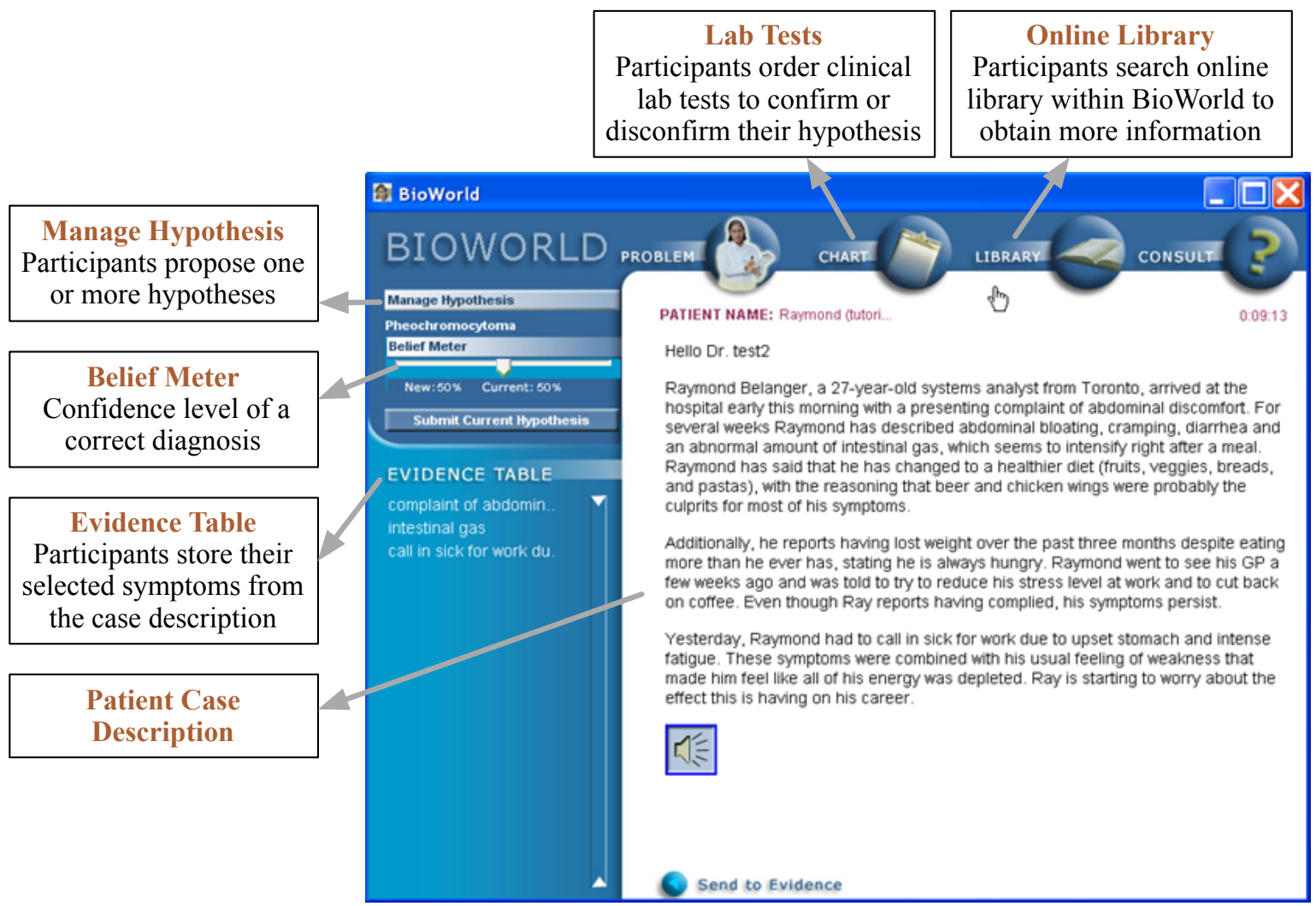

Figure 1. The interface of BioWorld

In this study, we chose the Amy case based mainly upon students' prior knowledge. The Amy case was created by a content expert and validated by two other experts who had expertise in medicine and learning sciences. The correct diagnosis for the virtual patient case was Diabetes Mellitus (type I).

\subsection{Procedure}

A training session was provided to the students to familiarize themselves with the BioWorld system prior to the experiment. Immediately afterward, students were asked to diagnose the virtual patient independently. Specifically, students performed the task individually in a lab class where they could ask research assistants for help if they encountered technical issues. Students' facial expressions were video recorded while they solved the case. Participants' problem-solving traces were automatically captured by the log files, which included the timestamp and duration of each activity. It took approximately 40 minutes for participants to complete the study.

\subsection{Performance, SRL, and emotion measures}

Diagnostic performance referred to the extent of evidence match between the participant's and an expert's solutions. In particular, an expert's solution was pre-configured in the BioWorld platform. Therefore, the participant's solution was automatically assessed in terms of evidence match with the expert's solution as soon as he/she submitted a final diagnosis. As such, performance is a continuous variable. Log files were used to categorize clinical reasoning behaviours (e.g., order lab tests, prioritize evidence items) into three SRL phases based on the coding scheme developed by Li et al. (2018) (see Table 1). 
Table 1

The coding scheme for analyzing SRL behaviors of clinical reasoning

\begin{tabular}{|c|c|c|c|}
\hline SRL Phases & Clinical Behaviours & Code & Description \\
\hline Forethought & $\begin{array}{l}\text { Collecting evidence } \\
\text { items }\end{array}$ & $\mathrm{CO}$ & $\begin{array}{l}\text { Collecting evidence items from the patient } \\
\text { description by recalling one's prior knowledge } \\
\text { pertaining to the symptoms }\end{array}$ \\
\hline \multirow{3}{*}{ Performance } & $\begin{array}{l}\text { Raising/Managing } \\
\text { hypotheses }\end{array}$ & RA & $\begin{array}{l}\text { Outlining a single or multiple diagnostic } \\
\text { hypothesis based on the collected evidence } \\
\text { items }\end{array}$ \\
\hline & Adding tests & $\mathrm{AD}$ & Conducting medical lab tests \\
\hline & Searching library & SE & $\begin{array}{l}\text { Searching for particular information in the } \\
\text { library for additional explanations }\end{array}$ \\
\hline \multirow{4}{*}{ Self-Reflection } & $\begin{array}{l}\text { Categorizing } \\
\text { evidence/results }\end{array}$ & $\mathrm{CA}$ & $\begin{array}{l}\text { Checking the relevance of evidence items and } \\
\text { lab test results towards a specific hypothesis } \\
\text { (i.e., whether the evidence/tests in support, } \\
\text { against, or neutral of one hypothesis) }\end{array}$ \\
\hline & $\begin{array}{l}\text { Linking } \\
\text { evidence/results }\end{array}$ & LI & $\begin{array}{l}\text { Justifying the probability of a hypothesis being } \\
\text { correct to the disease }\end{array}$ \\
\hline & $\begin{array}{l}\text { Prioritizing } \\
\text { evidence/results }\end{array}$ & PR & $\begin{array}{l}\text { Ranking evidence items and lab test results } \\
\text { according to their importance to a hypothesis }\end{array}$ \\
\hline & $\begin{array}{l}\text { Summarization for } \\
\text { final diagnosis }\end{array}$ & SU & $\begin{array}{l}\text { Making the final diagnosis by writing a } \\
\text { summarization }\end{array}$ \\
\hline
\end{tabular}

Students' emotions expressed in each SRL phase were analysed using FaceReader 6 software (Den Uyl, Van Kuilenburg, \& Lebert, 2005). FaceReader is a facial expression recognition software that can classify students' facial expressions into one of the six basic emotions: happy, sad, angry, surprised, scared, and disgusted. In FaceReader, each emotion is expressed as a value between 0 and 1 for a target face. An expression is scored "dominant" when its intensity is significantly higher than that of all others. The output of FaceReader consists of two columns of information, i.e., timestamps and corresponding emotional states. As such, researchers can identify the duration of an emotional state and when it changes to a different emotion over the course of problem-solving. Given that FaceReader detects emotions at a fine-grained temporal resolution in an automated fashion, it provides researchers a unique tool to examine the frequency of emotions and emotion variability that other emotion measures cannot afford. The software analyses facial expressions with an accuracy of 95\% (Noldus Information Technology, 2015). The accuracy of this software has also been verified by several researchers in empirical psychological studies (Chentsova-Dutton \& Tsai, 2010; Harley et al., 2015). The frequency of emotions and emotion variability were calculated for each SRL phase. Specifically, we calculated the frequency of emotions by dividing the number of emotions an individual expressed in a specific SRL phase by the length of that phase. Moreover, we calculated the probability distribution of the six types of basic emotions for each of the three SRL phases. For instance, the probability of happiness is .25 if an individual experiences 100 emotions in total in the performance phase, among which 25 are happy. Afterward, emotion variability was calculated using the Shannon entropy formula (Jack, Garrod, \& Schyns, 2014):

$$
h\left(p_{1}, \ldots, p_{\mathrm{a}}\right)=-\sum_{i=1}^{a} p \mathrm{i} \log (p \mathrm{i})
$$


where $p \mathrm{i}$ is the probability of emotional state $i$ appearing in a certain stream of emotions. Shannon entropy provides a mathematical way to quantify the randomness of emotional states. The minimum value of emotion variability is zero, indicating that students' emotion never varies. The maximum value of emotional variability is 2.58 (the base-2 logarithm of the six possible emotions) indicating the highest variability.

\subsection{Data Analysis}

To address our research questions, we applied the assessment method of Averaging over Orderings proposed by Lindeman, Merenda, and Gold (LMG) (Lideman, Merenda, \& Gold, 1980) to compare the relative importance of the six predictive variables (i.e., emotion frequency - forethought, emotion frequency - performance, emotion frequency - reflection, emotion variability - forethought, emotion variability - performance, emotion variability - reflection) in predicting diagnostic performance. In particular, the LMG approach examines the proportion of variance explained by each variable of interest, considering both its direct effect and its effect when combined with the other variables. The LMG method also takes the dependence on orderings into consideration instead of adding the predictors in the model randomly (Lideman et al., 1980). The formulae denoted as LMG can be written as:

$$
\begin{aligned}
& \operatorname{LMG}\left(x_{k}\right)=\frac{1}{p !} \sum_{r \text { permutation }} \operatorname{seqR}^{2}\left(\left(\left\{x_{k}\right\}\right) \mid r\right) \\
& \operatorname{seqR}^{2}\left(\left(\left\{x_{k}\right\}\right) \mid S_{k}(r)\right)=R^{2}\left(\left\{x_{k}\right\} \cup S_{k}(r)\right)-R^{2}\left(S_{k}(r)\right)
\end{aligned}
$$

$S_{k}(r)$ represents the set of predictors entered the linear model before the predictor $x_{k}$ in the order $r$, while seq $R^{2}\left(\left(\left\{x_{k}\right\}\right) \mid S_{k}(r)\right)$ represents the portion of $R^{2}$ (sum of squares) allocated to predictor $x_{k}$ in the order $r$. For example, for the six explanatory variables $(p=6)$, there are 720 different orderings $(6 !=6 \times 5 \times 4 \times 3 \times 2 \times 1=720)$ and 720 different estimations, i.e., sequential sum of squares. The relative importance of the explanatory variable is the mean of the 720 estimations.

A review of different methods for assessing relative importance suggests that LMG is one of the most computer-intensive methods and is widely adopted by researchers (Grömping, 2006). To assess if a variable is clearly different from the others in terms of relative importance, we used bootstrap percentile CIs (confidence intervals) to assess the variability of the estimates. The confidence intervals for differences show if differences in contributions can be considered statistically significant. Specifically, 1000 bootstrap samples were requested in analyses to estimate the CIs for differences between relative contributions.

\section{Results}

\subsection{Descriptive Analysis}

The mean values of the frequency of emotions, emotion variability, and performance were shown in Table 2. We also examined the bivariate correlations between those variables. The results of the descriptive analysis showed that students were generally in neutral affective states in the forethought phase, while they expressed their emotions strongly in the performance and self-reflection phases. The frequency of emotions in the performance phase highly correlated with the frequency of emotions in the self-reflection phase. Moreover, the emotion variability in the performance phase highly correlated with that in the self-reflection phase. Regarding the correlations between emotional variables and performance, the frequency of emotions in the self-reflection phase was only the factor that significantly associated with performance. 
Table 2

Descriptive analysis of the frequency of emotions, emotion variability, and performance

\begin{tabular}{lccccccccc}
\hline Variable & Mean & $S D$ & 1 & 2 & 3 & 4 & 5 & 6 & 7 \\
\hline 1.Emotion-Forethought & 2.10 & 1.95 & - & & & & & & \\
2.Emotion-Performance & 11.64 & 7.60 & .12 & - & & & & \\
3.Emotion-Self-reflection & 6.38 & 6.57 & .39 & $.46^{*}$ & - & & & \\
4.Emotion Variability-Forethought & 1.23 & .50 & .25 & .19 & .17 & - & & \\
5.Emotion Variability-Performance & 1.56 & .41 & .24 & .13 & .24 & .24 & - & & \\
6.Emotion Variability-Self-reflection & 1.41 & .48 & .02 & .17 & .32 & .21 & $.68^{* *}$ & - & \\
7.Performance & 53.00 & 13.82 & .07 & .04 & $.49^{*}$ & -.36 & -.11 & -.08 & - \\
\hline
\end{tabular}

Note: The values in the last seven columns are Pearson correlations. The symbols * and $* *$ indicates that correlation is significant at the .05 and .01 levels (2-tailed), respectively.

In addition, we calculated the average durations of the three SRL phases and the mean values of the frequency of the six basic emotions, for the sake of helping readers gain a comprehensive understanding of students' emotional experience (see Table 3). In general, the frequencies of all the six types of emotions increased from the forethought phase to the performance phase, and then decreased as students moved from the performance phase to the self-reflection phase.

Table 3

The average duration of the three SRL phases and the mean values of the frequency of the six basic emotions

\begin{tabular}{lcccccccc}
\hline & Duration & $S D$ & Angry & $\begin{array}{c}\text { Disguste } \\
\mathrm{d}\end{array}$ & $\begin{array}{c}\text { Happ } \\
\mathrm{y}\end{array}$ & $\begin{array}{c}\text { Surprise } \\
\mathrm{d}\end{array}$ & Sad & Scared \\
\hline Forethought & 3.15 & 1.99 & 0.84 & 0.31 & 0.14 & 0.62 & 0.07 & 0.10 \\
Performance & 17.06 & 7.83 & 5.63 & 2.18 & 0.99 & 2.20 & 0.31 & 0.33 \\
Self-reflection & 8.76 & 5.53 & 2.68 & 0.94 & 0.62 & 1.87 & 0.10 & 0.16 \\
\hline
\end{tabular}

Note: The first two columns show the average duration of each SRL phase and corresponding standard deviation of the durations. The values in the remaining columns are the mean frequencies of a specific emotion, i.e., the number of a specific emotion per minute.

\subsection{Relative Importance Analysis}

The total proportion of variance in students' diagnostic performance explained by the six emotion-related predictors was $50.67 \%$. According to Cohen (1988), the effect size is large (>.26). Considering that clinical reasoning is an active and constructive process whereby students need to manipulate their cognitive, metacognitive, affective, and motivational aspects of problem-solving, this result has demonstrated the crucial roles of the frequency of emotions and emotion variability in the context of diagnosing patients. In particular, the results in Table 4 showed that the frequency of emotions in the forethought and performance phases of self-regulated learning negatively predicted performance ( $r=-.66$ and $r=-.32$, respectively), while the frequency of emotions in the self-reflection phase positively predicted performance $(r=1.55)$. In general, emotion variability negatively predicted performance regardless of which SRL phases it was tied to. 
Table 4

Relative importance of emotion and emotion variability in predicting performance

\begin{tabular}{lccc}
\hline Variable & $R^{2}(\mathrm{lmg})$ & $95 \% \mathrm{CI}$ & $\begin{array}{c}\text { Average Coefficient } \\
\text { (Standardized coefficient) }\end{array}$ \\
\hline Emotion-Forethought & .0114 & {$[.0024, .1322]$} & $-.66(-.09)$ \\
Emotion-Performance & .0172 & {$[.0052, .1076]$} & $-.32(-.17)$ \\
Emotion-Self-reflection & .3071 & {$[.0646, .6325]$} & $1.55(.74)$ \\
Emotion variability-Forethought & .1458 & {$[.0258, .3371]$} & $-10.70(-.39)$ \\
Emotion variability-Performance & .0102 & {$[.0061, .1664]$} & $-.70(-.02)$ \\
Emotion variability-Self-reflection & .0150 & {$[.0106, .2738]$} & $-5.33(-.19)$ \\
\hline
\end{tabular}

Note: Emotion-Forethought, Emotion-Performance, and Emotion-Self-reflection refer to the frequency of emotions in the forethought, performance, and self-reflection phase, respectively; lmg, also known as $R^{2}$, is an index of the relative importance of predictive variables in predicting the outcome variable; $95 \%$ CI $=95 \%$ confidence interval. Similar to conventional regression coefficients, average coefficients indicate the relationship between each of the predictors and a dependent variable. However, the predictors must be uncorrelated in regression models, while the predictors do not have to meet the multicollinearity assumption when estimating their coefficients with the LMG method. The LMG method takes the dependence of the predictors into account and produces what are called average coefficients.

Moreover, the results in Table 4 and Figure 2 indicated that the frequency of emotions in the self-reflection phase $\left(R^{2}=.3071\right)$ and emotion variability in the forethought phase $\left(R^{2}=.1458\right)$ were the two most important factors in predicting performance. The confidence intervals for differences between relative contributions, as shown in Table 5, revealed that the difference in the frequency of emotion between the self-reflection phase and the forethought phase, as well as the difference between the selfreflection phase and the performance phase, were statistically significant. Specifically, the frequency of emotions in the self-reflection phase was significantly more important than that of the other two SRL phases in predicting clinical reasoning performance. Furthermore, there were no significant differences between the three SRL phases in terms of students' emotion variability. There were also no significant differences between the frequency of emotions and emotion variability regardless of the SRL phases.

\section{Relative importances for ExpertMatch with $95 \%$ bootstrap confidence intervals}

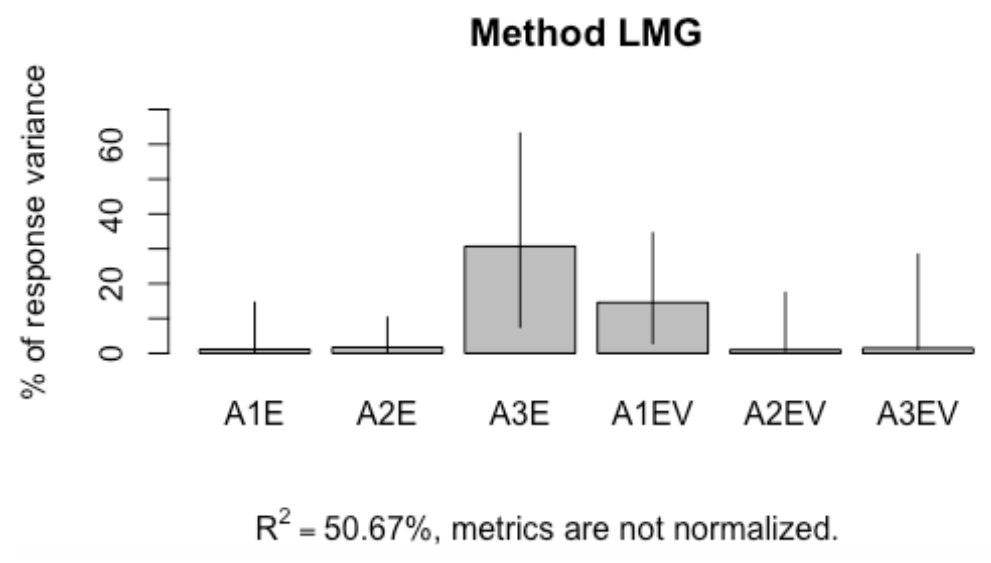

Figure 2. Visualization of the relative importance of the factors in predicting performance. Note: A1E, $\mathrm{A} 2 \mathrm{E}$, and $\mathrm{A} 3 \mathrm{E}$ refer to the frequency of emotions in the forethought, performance, and self-reflection phase, respectively; A1EV, A2EV, and A3EV refer to emotion variability in the forethought, 
performance, and self-reflection phase, respectively; Diagnostic performance was indicated by the evidence match between students' and experts' solutions. The total proportion of variance explained by the model was $50.67 \%$.

Table 5

Bootstrap confidence intervals for differences between relative contributions

\begin{tabular}{lcc}
\hline & Difference & $95 \%$ CI \\
\hline A1E-A2E & -.0058 & {$[-.0860, .1014]$} \\
A1E-A3E & $-.2958 *$ & {$[-.6101,-.0245]$} \\
A1E-A1EV & -.1344 & {$[-.3207, .0548]$} \\
A1E-A2EV & .0012 & {$[-.1366, .0939]$} \\
A1E-A3EV & -.0036 & {$[-.2606, .0988]$} \\
A2E-A3E & $-.2900 *$ & {$[-.6071,-.0250]$} \\
A2E-A1EV & -.1286 & {$[-.3051, .0266]$} \\
A2E-A2EV & .007 & {$[-.1302, .0661]$} \\
A2E-A3EV & .0022 & {$[-.2483, .0554]$} \\
A3E-A1EV & .1614 & {$[-.1887, .5695]$} \\
A3E-A2EV & .2970 & {$[-.0410, .5905]$} \\
A3E-A3EV & .2921 & {$[-.1788, .5862]$} \\
A1EV-A2EV & .1356 & {$[-.0802, .2977]$} \\
A1EV-A3EV & .1307 & {$[-.1643, .2821]$} \\
A2EV-A3EV & -.0049 & {$[-.2028, .0997]$} \\
\hline
\end{tabular}

Note: * indicates that CI for difference does not include 0. A1E, A2E, and A3E refer to the frequency of emotions in the forethought, performance, and self-reflection phase, respectively; A1EV, A2EV, and $\mathrm{A} 3 \mathrm{EV}$ refer to emotion variability in the forethought, performance, and self-reflection phase, respectively

\section{Discussion}

This research revealed that the frequency of emotions in both the forethought and performance phases of SRL negatively predicted performance in clinical reasoning, while the frequency of emotions in the self-reflection phase positively predicted performance. A potential explanation is that a high frequency of emotions in the forethought and performance phases may interfere with students' cognition and motivation. It is highly possible that students who failed to regulate their emotions in these two phases could not concentrate on their decision-making and executing behaviours. An exception is the frequency of emotions in the self-reflection phase, a period when students receive and make sense of the collected evidence and test results. According to Schutz and Davis (2000), emotions in the selfreflection phase can be due to cognitive appraisals about the progress of their diagnosis, which influence how students adjust their problem-solving strategies and final performance. Moreover, the work of BenEliyahu (2019) offered another explanation for these findings. Specifically, students' affective inclination moderates how activity emotions are linked to performance. However, there is a possibility that the moderation effect declines as learning or problem-solving process unfolds. Therefore, the effect of affective inclination on the emotions in the self-reflection phase is different from the forethought and performance phases. 
In addition, this study found that emotion variability had a negative influence on diagnostic performance, regardless of SRL phases it was tied to. This finding highlights the importance of keeping a stable emotional state to guarantee high performance, which has been corroborated by previous research (Thompson et al., 2017). This finding also has implications for teaching and learning regarding emotion regulation. As argued by Ben-Eliyahu (2019), "teaching learners to regulate emotions to facilitate potential benefits during intellectual work can and should be an explicit target during learning" (p. 86). It must be noted that emotions occur in specific situations as responses to the features of external environment and internal appraisals (Gross, 2013). The finding of this study is influenced by the features of clinical reasoning and the unique characteristics of medical students. Therefore, this finding needs to be interpreted appropriately in other contexts.

Moreover, the frequency of emotions and emotion variability were found to jointly affect diagnostic performance. This result is not surprising since prior research has found both variables are important when considered independently (Barrett, 2006; Gruber et al., 2013; Kashdan \& Rottenberg, 2010; McConnell et al., 2016; Thompson et al., 2017). A unique contribution of this study is that we examined the two variables simultaneously in one study, which could help gain a better understanding of the role of emotions in learning. In addition to the novelty of the research context, this study also provided methodological insights regarding the measurement of emotions at a fine-grained temporal resolution and the analysis of emotion variability. Further insights are that the two most important factors in predicting performance were the frequency of emotions in the self-reflection phase and emotion variability in the forethought phase. The frequency of emotions in the self-reflection phase may be due to students' awareness that their final diagnosis is correct. Variability in the forethought phase may be due to the open consideration of all possibilities for a diagnosis, making students less stable or secure in their ability and perhaps more emotionally variable. However, future research is needed to shed light on how and why these two factors affect performance.

In sum, findings from this research alert medical researchers to the important role that emotion plays in clinical reasoning. Medical teachers and students alike should be aware of the close ties between the frequency of emotions, emotion variability, and diagnostic performance. Moreover, findings from this research resonate with the call for more studies on emotion-related regulation in the context of clinical reasoning. Medical students would benefit from the training programs regarding how to redirect, control, and modify emotional arousals to perform adaptively in emotionally arousing situations. For instance, this study suggested that restraining substantial changes in emotions across the whole diagnostic process and reappraising emotions in the early stages of clinical reasoning (i.e., forethought and performance phase) may lead to better diagnostic reasoning outcomes. This finding partially aligns with prior work on emotion regulation that suppression was found positively related to positive deactivated emotions in certain contexts, and positive deactivated emotions (e.g., relief) were indicators of high performance (Ben-Eliyahu \& Linnenbrink-Garcia, 2013). It is noteworthy that researchers' interest in emotion-related regulation has grown enormously in recent years, and there is substantial research on emotion regulation strategies (Eisenberg et al., 2018; LeBlanc, Essau, \& Ollendick, 2017). Such strategies include, but are not limited to, changing how one appraises a situation, regulating the demands of familiar settings, selecting adaptive response alternatives, encoding of internal emotion cues, and accessing to coping resources (Siemer et al., 2007). It would be fruitful for future studies to examine which types of emotion regulation strategies are effective in clinical reasoning, taking the particular context and the characteristics of individuals into account.

\section{Conclusion}

This study examined the relative importance of the frequency of emotions and emotion variability in the three SRL phases (i.e., forethought, performance, and self-reflection) in predicting diagnostic performance. We found that both the frequency of emotions and emotion variability matter to students' performance, and they functioned differently in the clinical reasoning process. This study helps shift the focus of research from the role of emotions in learning alone, towards the joint effects of 
emotions and emotion variability on performance. This study has also methodological insights with regards to the analysis of emotion variability and the relative importance of different emotion features.

It is important to note that this study is not without limitations. We examined the influence of the frequency of emotions in each SRL phase on diagnostic performance rather than the frequency of each specific emotion, for example, happy and angry. It is possible that one type of emotion has greater predictive power than another emotion. In addition, students experience a wide range of emotions in academic settings that may go beyond the scope of basic emotions. Therefore, it is necessary to advance methodological innovations in capturing different categories of emotions in situ for future studies. Furthermore, students may have a mixed emotional state, whereas we recognized one dominant emotion at a given time due to the constraints of emotion measures. Lastly, a larger cohort of medical students is needed to verify the generalizability of our findings as they diagnose patients with different levels of complexities.

As a closing remark, this study lays the foundation for future advances in emotion-related study designs since the introduction of emotion variability leaves many questions unanswered and shows promise for new research directions. For example, does affective inclination affect emotion variability (Ben-Eliyahu, 2019)? What are the differences between emotion regulation and the regulation of emotion variability? Are appraisals sufficient causes of emotion variability? How does emotion variability affect learning in the long run? It would also be interesting to take the valence of emotions into account when investigating the frequencies and variabilities of emotions. For instance, the frequency of positive emotions or the variability of negative emotions may tell an in-depth story about students' performance differences. Another related direction for future research is to assess the relative importance of the valence, frequency, and variability of emotions to learning performance. In addition, it is promising to examine the variability in emotional intensity and its relationships with cognition, metacognition, and learning performance. 


\section{Key points}

- This study is one of the first to explore the variability aspect of emotions in SRL (selfregulated learning), which has the potential to open new research directions.

- We identified students' real-time emotions from their facial expressions as they diagnosed virtual patients in a computer-simulated environment.

6. We calculated the frequency of emotions and emotion variability at each SRL phase: forethought, performance, and self-reflection.

- We used the assessment method of Averaging over Orderings to examine the joint effects of emotion and emotion variability on clinical reasoning performance.

- We found that both emotion and emotion variability affected students' performance and they functioned differently in the SRL process. Emotion variability negatively predicted performance regardless of which SRL phases it was tied to.

\section{Acknowledgments}

This research was funded by the Fonds de recherche du Québec - Société et culture (FRQSC) and the Social Sciences and Humanities Research Council of Canada (SSHRC)

\section{References}

Ahmed, W., van der Werf, G., Kuyper, H., \& Minnaert, A. (2013). Emotions, self-regulated learning, and achievement in mathematics: A growth curve analysis. Journal of Educational Psychology, 105(1), 150-161. http://dx.doi.org/10.1037/a0030160

Artino, A. R., Hemmer, P. A., \& Durning, S. J. (2011). Using self-regulated learning theory to understand the beliefs, emotions, and behaviors of struggling medical students. Academic Medicine, 86(10), S35-S38. https://doi.org/10.1097/ACM.0b013e31822a603d

Artino, A. R., Holmboe, E. S., \& Durning, S. J. (2012). Can achievement emotions be used to better understand motivation, learning, and performance in medical education? Medical Teacher, 34(3), 240-244. https://doi.org/10.3109/0142159X.2012.643265

Barrett, L. F. (2006). Solving the emotion paradox: categorization and the experience of emotion. Personality and Social Psychology Review, 10(1), 20-46. https://doi.org/10.1207/s15327957pspr1001_2

Barrett, L. F. (2009). Variety is the spice of life: A psychological construction approach to understanding variability in emotion. Cognition and Emotion, 23(7), 1284-1306. https://doi.org/10.1080/02699930902985894

Ben-Eliyahu, A. (2019). Academic emotional learning: A critical component of self-regulated learning in the emotional learning cycle. Educational Psychologist, 54(2), 84-105. https://doi.org/10.1080/00461520.2019.1582345

Ben-Eliyahu, A., \& Linnenbrink-Garcia, L. (2013). Extending self-regulated learning to include selfregulated emotion strategies. Motivation and Emotion, 37(3), 558-573. https://doi.org/10.1007/s11031-012-9332-3

Ben-Eliyahu, A., \& Linnenbrink-Garcia, L. (2015). Integrating the regulation of affect, behavior, and cognition into self-regulated learning paradigms among secondary and post-secondary students. Metacognition and Learning, 10(1), 15-42. https://doi.org/10.1007/s11409-014-9129-8

Bi, J. (2012). A review of statistical methods for determination of relative importance of correlated predictors and identification of drivers of consumer liking. Journal of Sensory Studies, 27(2), 87101. https://doi.org/10.1111/j.1745-459X.2012.00370.x

Chentsova-Dutton, Y. E., \& Tsai, J. L. (2010). Self-focused attention and emotional reactivity: The role of culture. Journal of Personality and Social Psychology, 98(3), 507-519. https://doi.org/10.1037/a0018534 
Cohen, J. (1988). Statistical power analysis for the behavioral sciences (2nd ed.). Lawrence Erlbaum Associates.

Den Uyl, M., Van Kuilenburg, H., \& Lebert, E. (2005). FaceReader: an online facial expression recognition system. In Proceedings of the 5th International Conference on Methods and Techniques in Behavioral Research (Vol. 2005, pp. 589-590).

Eisenberg, N., Spinrad, T. L., \& Valiente, C. (2018). Emotion-related self-regulation and children's social, psychological, and academic functioning. In Diversity in harmony-Insights from psychology: Proceedings of the 31st International Congress of Psychology (pp. 268-295). Wiley Online Library.

Ekman, P. (1992). An Argument for Basic Emotions. Cognition and Emotion, 6(3-4), 169-200. https://doi.org/10.1080/02699939208411068

Grömping, U. (2006). Relative importance for linear regression in R: The package relaimpo. Journal of Statistical Software, 17(1), 1-27. https://doi.org/10.18637/jss.v017.i01

Gross, J. J. (1998). The emerging field of emotion regulation: An integrative review. Review of General Psychology, 2(3), 271-299. https://doi.org/10.1037/1089-2680.2.3.271

Gross, J. J. (2013). Emotion regulation: Conceptual and empirical foundations. In J. J. Gross (Ed.), Handbook of Emotion Regulation (2nd ed., pp. 3-20). Guilford Publications.

Gruber, J., Kogan, A., Quoidbach, J., \& Mauss, I. B. (2013). Happiness is best kept stable: Positive emotion variability is associated with poorer psychological health. Emotion, 13, 1-6. http://dx.doi.org/10.1037/a0030262

Harley, J. M., Bouchet, F., Hussain, M. S., Azevedo, R., \& Calvo, R. (2015). A multi-componential analysis of emotions during complex learning with an intelligent multi-agent system. Computers in Human Behavior, (48), 615-625. https://doi.org/10.1016/j.chb.2015.02.013

Izard, C. E. (2007). Basic emotions, natural kinds, emotion schemas, and a new paradigm. Perspectives on Psychological Science, 2(3), 260-280. https://doi.org/10.1111/j.1745-6916.2007.00044.x

Jack, R. E., Garrod, O. G. B., \& Schyns, P. G. (2014). Dynamic facial expressions of emotion transmit an evolving hierarchy of signals over time. Current Biology, 24(2), 187-192. https://doi.org/10.1016/j.cub.2013.11.064

John, O. P., Gross, J. J. (2006). Individual differences in emotion regulation. In J. J. Gross (Ed.), Handbook of Emotion Regulation (1st ed., pp. 351-372). Guilford Publications.

Kashdan, T. B., \& Rottenberg, J. (2010). Psychological flexibility as a fundamental aspect of health. Clinical Psychological Review, 30(7), 865-878. https://doi.org/10.1016/j.cpr.2010.03.001

Lajoie, S. P. (2009). Developing professional expertise with a cognitive apprenticeship model: examples from avionics and medicine. In K. A. Ericsson (Ed.), Development of Professional Expertise: Toward Measurement of Expert Performance and Design of Optimal Learning Environments (pp. 61-83). New York: Cambridge University Press.

Lajoie, S. P., Zheng, J., \& Li, S. (2018). Examining the role of self-regulation and emotion in clinical reasoning: Implications for developing expertise. Medical Teacher, 40(8), 842-844. https://doi.org/10.1080/0142159X.2018.1484084

Lajoie, S. P., Zheng, J., Li, S., Jarrell, A., \& Gube, M. (2019). Examining the interplay of affect and self regulation in the context of clinical reasoning. Learning and Instruction, 101219. https://doi.org/10.1016/j.learninstruc.2019.101219

LeBlanc, S., Essau, C. A., \& Ollendick, T. H. (2017). Emotion regulation: An introduction. In C. A. Essau, S. Leblanc, \& T. H. Ollendick (Eds.), Emotion regulation and psychopathology in children and adolescents. (1st ed., pp. 3-17). Oxford: Oxford University Press. https://doi.org/10.1093/med:psych/9780198765844.003.0001

Li, S., \& Lajoie, S. P. (2021). Cognitive engagement in self-regulated learning: An integrative model. European Journal of Psychology of Education, 1-20. https://doi.org/10.1007/s10212-021-00565$\mathrm{X}$

Li, S., Zheng, J., Poitras, E., \& Lajoie, S. (2018). The allocation of time matters to students' performance in clinical reasoning. In R. Nkambou, R. Azevedo, \& J. Vassileva (Eds.), Lecture notes in computer sciences (pp. 110-119). Springer International Publishing AG, part of Springer Nature. https://doi.org/10.1007/978-3-319-91464-0_11 
Lideman, R., Merenda, P., \& Gold, R. (1980). Introduction to bivariate and multivariate analysis scott. Scott Foresman: Glenview, IL, USA.

McConnell, M. M., \& Eva, K. W. (2012). The role of emotion in the learning and transfer of clinical skills and knowledge. Academic Medicine, 87(10), 1316-1322. https://doi.org/10.1097/ACM.0b013e3182675af2

McConnell, M. M., Monteiro, S., Pottruff, M. M., Neville, A., Norman, G. R., Eva, K. W., \& Kulasegaram, K. (2016). The impact of emotion on learners application of basic science principles to novel problems. Academic Medicine, 91(11), 58-63. https://doi.org/10.1097/ACM.0000000000001360

Noldus Information Technology. (2015). Reference manual: FaceReader version 6.1. Wageningen, The Netherlands: Noldus Information Technology International Headquarters.

Oliver, M. N. I., \& Simons, J. S. (2004). The affective lability scales: Development of a short-form measure. Personality and Individual Differences, 37, 1279-1288. https://doi.org/10.1016/j.paid.2003.12.013

Pekrun, R., Goetz, T., Titz, W., \& Perry, R. P. (2002). Academic emotions in students' self-regulated learning and achievement: A program of qualitative and quantitative research. Educational Psychologist, 37(2), 91-105. https://doi.org/10.1207/S15326985EP3702_4

Pekrun, R., \& Stephens, E. J. (2010). Achievement emotions: A control-value approach. Social and Personality Psychology Compass, 4(4), 238-255. https://doi.org/10.1111/j.17519004.2010.00259.x

Pintrich, P. R. (2000). The role of goal orientation in self-regulated learning. In M. Boekaerts, P. R. Pintrich, \& M. Zeidner (Eds.), Handbook of Self-Regulation (1st ed., pp. 451-502). San Diego, CA: US: Academic Press.

Scherer, K. R. (2005). What are emotions? And how can they be measured? Social Science Information, 44(4), 695-729. https://doi.org/10.1177/0539018405058216

Schutz, P. A., \& Davis, H. A. (2000). Emotions and Self-Regulation During Test Taking. Educational Psychologist, 35(4), 243-256. https://doi.org/10.1207/S15326985EP3504_03

Siemer, M., Mauss, I., \& Gross, J. J. (2007). Same situation-different emotions: How appraisals shape our emotions. Emotion, 7(3), 592-600. https://doi.org/10.1037/1528-3542.7.3.592

Thompson, R. J., Boden, M. T., \& Gotlib, I. H. (2017). Emotional variability and clarity in depression and social anxiety. Cognition and Emotion, 31(1), 98-108. https://doi.org/10.1080/02699931.2015.1084908

Tracy, J. L., \& Randles, D. (2011). Four models of basic emotions: A review of Ekman and Cordaro, Izard, Levenson, and Panksepp and Watt. Emotion Review, 3(4), 397-405. https://doi.org/10.1177/1754073911410747

Xu, S., Martinez, L. R., Van Hoof, H., Eljuri, M. I., \& Arciniegas, L. (2016). Fluctuating emotions: relating emotional variability and job satisfaction. Journal of Applied Social Psychology, 46, 617 626. https://doi.org/10.1111/jasp.12390

Zimmerman, B. J. (2000). Attaining self-regulation: A social cognitive perspective. In M. Boekaerts, P. R. Pintrich, \& M. Zeidner (Eds.), Handbook of Self-Regulation (1st ed., pp. 13-39). San Diego (CA): Academic Press. https://doi.org/10.1016/B978-012109890-2/50031-7 